\title{
Interference Mitigation Using Co-operative Localization with Co-operative sensing and relays for Cognitive Radio Networks
}

\author{
${ }^{1}$ G.Samuelraj Chrysolite, ${ }^{2}$ K.Gunaseelan \\ ${ }^{1,2}$ Department of Electronics and Communication Engineering \\ College of Engineering, Anna University, Guindy,Chennai, India \\ g.samuelraj@gmail.com,guna_2012@annauniv.edu
}

\begin{abstract}
A change in the transmission parameters are never entertained when it comes to a network where the transmission power is always wanted to be kept a constant for consistent performance. However in a cognitive network scenario, interference mitigation can be improved by using cooperative relay which boosts the amplitude of the transmitted signal. An algorithm for relay selection is made use of for efficient interference mitigation. Moreover, a cooperative sensing mechanism can be incorporated in order to maximize the channel capacity of the cognitive user. This can be done for the benefit of the cognitive radios in a world which gives priority to the primary user transmissions. Hence, in this proposed algorithm, a complex system consisting of cooperative sensing mechanism and transmitting using a cooperative relay are explained.
\end{abstract}

Keywords: Cognitive users, cooperative localization, Cooperative Relay, Cooperative Sensing, Interference mitigation, RSS, Trilateration.

\section{INTRODUCTION}

The transmission power of primary transmitter cannot be adjusted in order to mitigate the interference at the primary receiver as one cannot order a licensed primary user to increase the power for any reason. Rather we can only lower the secondary transmission power as per the situation for interference mitigation

The cooperative relaying technique is based on the optimal stopping rule used to select the relay as in [1] and [2]. A major contribution to the cooperative localization technique comes from the "Least Square Cooperative Localization" as in [3] where the cooperative localization is done through the least square method of the singular value decomposition. A short survey of the range based localization algorithm is done in [4] which lists out some of the range based algorithms.. The trilateration method is explained in detail in [5]. The shadowing effects in the wireless channel is also dealt with as in [6].

Cooperative Relaying technique is used in this paper in order to enhance the signal quality after the signal has been transmitted from the primary transmitter before reception at the primary receiver. The algorithm for the same, has been proposed as in [1]. Here the relay selection plays a major role as it has a direct effect on the successful reception of the signal at the receiver, and also thereby affecting the interference mitigation.

Along with this relaying technique, the cooperative sensing part is also added in order to add a formidable advantage to the secondary users in network which wants to transmit. If the secondary users sense that there is no primary user transmitting through cooperative sensing, then the secondary user can transmit through the licensed spectrum with full power. This helps to improve the capacity of the transmission.

In the rest of the paper, a short recap of the interference mitigation by cooperative localization is presented in section II. Then in section III, cooperative relay selection is discussed followed by the cooperative sensing technique used. Then the overall algorithm and the pictorial representation of the proposed algorithm is explained. Then the inference from the proposed algorithm producing better EVM and Capacity for both cooperative sensing and relay are explained. Section IV shows the simulation results taken on the basis of performance analysis using MATLAB. Section V concludes the paper with future works and the references.

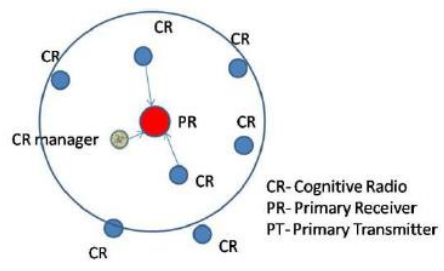

Figure 1. Cooperative localization with CR Manager

\section{INTERFERENCE MITIGATION BY COOPERATIVE LOCALIZATION}

A model for the cognitive radio network, with primary users and cognitive users involving both transmitters and receivers with the $\mathrm{CR}$ manager is shown in Fig. 1. Here the primary receiver is localized in order to reduce the interference due to the cognitive radios nearby using cooperative localization with the help of CR manager. Cooperative localization is a widely used concept for finding the location of a node with the help a priori information of the nodes around it. Out of many types of 
localization process, trilateration is used to find the location of the primary receiver.

Cooperation between the primary and cognitive radios is not possible and hence only cognitive radios are used for the localization process. The secondary user receivers are used as the cooperative users which receive the test beacon transmitted by the primary transmitter. To locate the primary receiver which is important than the former the secondary (cognitive) transmitters emit a test signal and the primary receiver which receive the bits transmitted send feedback to the respective transmitters with the RSSI information which is later formulated as the distance between those users. Then the nearest node is selected as CR manager which carries out the localization process after the formation of the local region. Here cooperation between the licensed users and the cognitive users are induced under mutual benefits (i.e the secondary or cognitive users will reduce interference based on the information provided by the primary receiver on reception of the signal from the secondary transmitters).

Now the distance between the primary and the cognitive radios and the cognitive radio's position (assumed to be known already) are used in the process of trilateration to find the position of the primary receiver as in [4] and [5].

A simple singular value decomposition is applied.

$$
(x, y)=\left(A^{T} A\right)^{-1} A^{T} b
$$

$(\mathrm{x}, \mathrm{y})$ - represents the $\mathrm{x}$ and $\mathrm{y}$ axis of the unknown primary receiver.

A - formed from coordinates of known secondary transmitters and the distance based on RSS (pseudo inverse taken).

B - a matrix created by forming circles around the secondary transmitters

Now the position of the primary receiver is used to find the exact distance from the cognitive radios to the primary radios. The distance found out using RSS and the coordinates are compared and reduction in the secondary transmission power is carried out whenever necessary.

\section{PROPOSED INTERFERENCE MITIGATION ALGORITHM} WITH IMPROVED CAPACITY

As per the proposed algorithm, it has three concepts,

- Cooperative Relay

- Cooperative Sensing

- Localization Process

\section{A. Cooperative Relay}

When the direct transmission from the transmitter to the receiver is error prone, an alternative path through another intermediate node is selected in order to provide better signal quality. Here the primary transmitter transmits and the reception at the primary receiver is not up to the standards of the RSS to be received, another path of transmission through another cognitive radio in the network is done which is known as relaying.
According to the proposed algorithm, the usual steps for finding out the position of the primary receiver using cooperative localization are carried out. Then both the distances are compared. Now when the there is a drastic difference between the two distances, one can say that the wireless transmission is dented by the attenuation factors in the wireless channel and also the NLOS problem. To solve this shadowing effect problem, the secondary transmitter power was reduced so that it does not cause sudden disruption in the transmission of the primary user. Here, along with reduction in secondary transmission power, the primary transmission power can be increased with use of cooperative relaying technique which in turn improves the performance of the system.

\section{B. Cooperative Relay Selection}

Therefore the selection of the cooperative relay is very important as it plays a significant role in the reduction of interference due to the secondary user transmissions. The selection of the cooperative relay is done using the optimal stopping rule as in [1].

Based on the optimal stopping rule, there are three major parameters for becoming the cooperative relay.

- Least distance from the receiver.

- High transmission rate

- Highest residue energy.

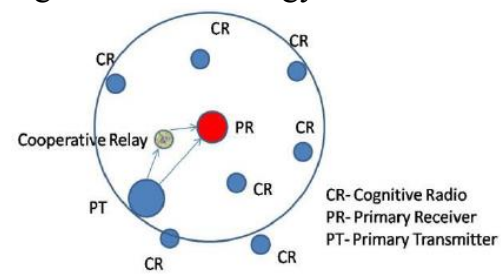

Figure 2. Cooperative Relaying Technique

The optimal stopping rule algorithm just monitors the cognitive radio nearby and evaluates them based on the above mentioned parameters and the node which gets the highest mark in that, is selected as the cooperative relay node.

\section{Cooperative Sensing}

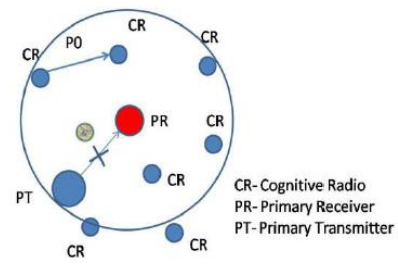

Figure 3. Defining the local region

Apart from the fact that we strive hard to improve the transmission of the primary users, the secondary users also need some assistance to transmit efficiently. Therefore we implement the cooperative sensing to check whether the primary transmitters are present or not.

In the proposed algorithm, only when the primary transmissions are present, interference mitigation is needed 
for the same. Hence when they are not present, at any point of time, the secondary user can transmit with full power. This in turn will increase the capacity of the secondary users and enable them to transmit more efficiently. Let the power of transmission when the primary transmissions are present be "P". Therefore through cooperative sensing when it finds that there are no current primary transmissions it can transmit at " $\mathrm{P}_{0}$ ". The cognitive users must sense the presence of primary transmissions collectively, through one of the spectrum sensing methods. Energy Detection method is the usual method used.

\section{Proposed Algorithm}

The aim of the proposed algorithm is to improve the EVM and capacity of the primary users through cooperative relaying technique. It also strives to improve the capacity of the secondary transmissions when there are no primary transmissions using the cooperative sensing mechanisms to find out whether the primary users are transmitting or not.

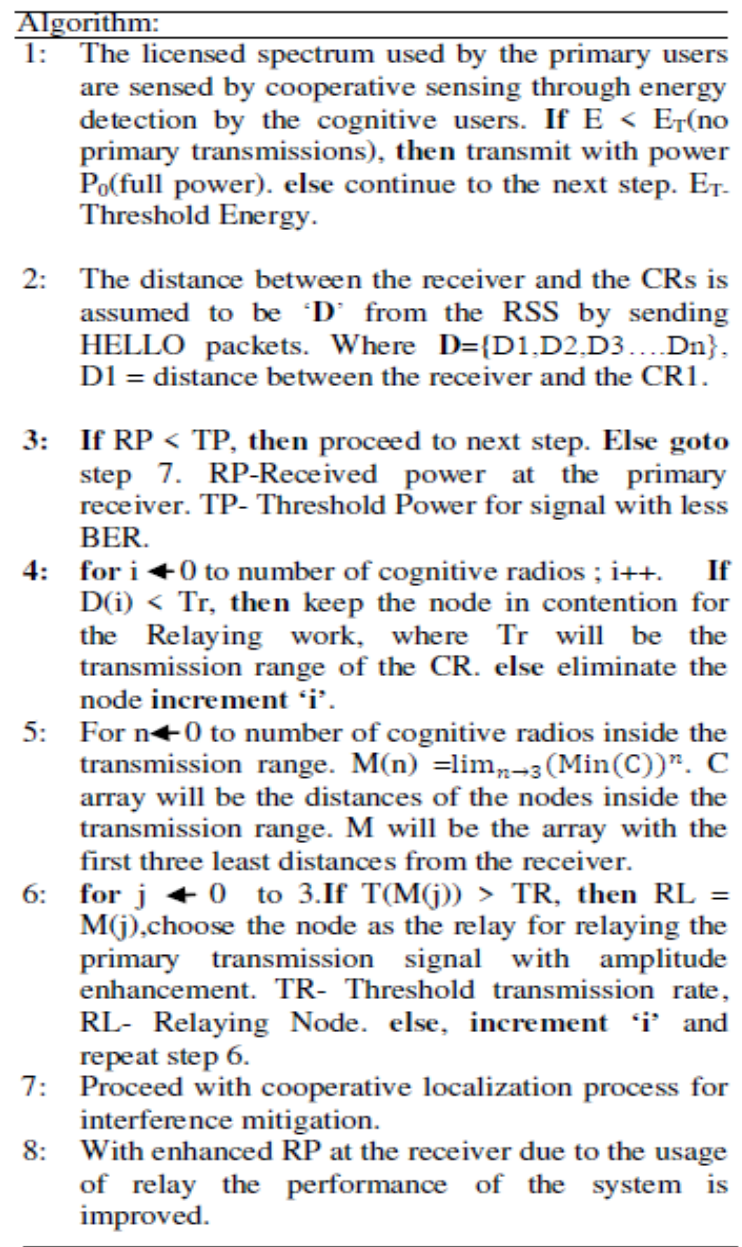

\section{E. Inferences}

According to the proposed algorithm, cooperative relaying technique and cooperative nesing are implemented.

1) From cooperative relaying

- When the RSS is weak at the primary receiver a relay is selected to propagate the primary signal with a increase in power at the relay node. Therefore a significant reduction in the error rate is noted.

- When the transmission power increases, the interference mitigates automatically leading to improved capacity.

2) From cooperative sensing

- This technique acts in favour of the secondary users when the primary users are not present.

- When there are no primary transmissions, the secondary users can transmit freely at power $\mathrm{P}_{0}$ (full power) which significantly increases the capacity.

- Cooperative sensing is used to avoid the hidden terminal problem and exposed terminal problem.

\section{SIMULATION RESULTS}

A general scenario is taken into consideration as shown in Fig. 5. A primary receiver which falls in the range of both the primary transmitter and the primary receiver is considered here.

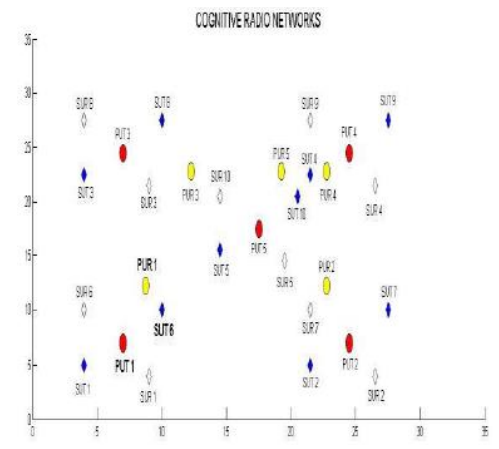

Figure 4. General Scenario

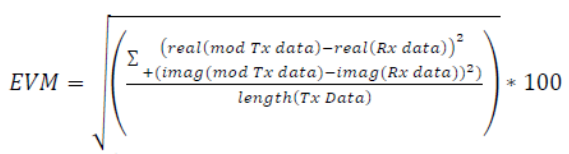

A graph plot for SINR vs EVM using cooperative relaying technique as in the proposed algorithm is plotted and the results are compared with the plot which does not use cooperative relaying technique. The same is done by calculating the channel capacity and plotting the SINR vs CAPACITY curve. When the cooperative sensing is implemented on the above scenario, the same graphs are 
plotted comparing the presence and the absence of the primary user.

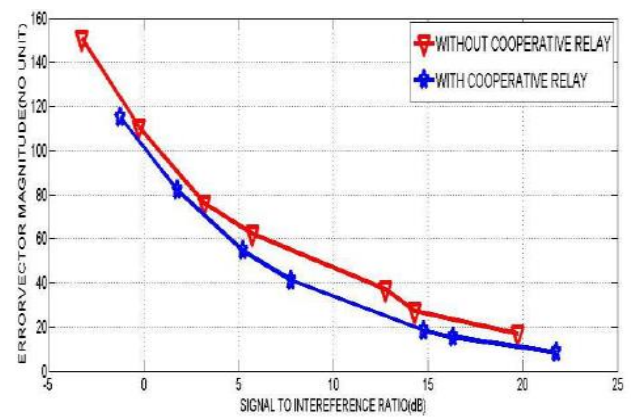

Figure 5. SINR VS ERROR VECTOR MAGNITUDE (at the primary receiver)

The EVM (Error Vector Magnitude) reduces to a certain extent because the relay node in between enhances the SNR at the primary receiver. Moreover we can achieve better SNR's with low transmission power as there is an intermediate node. A reduction of $10-15$ is seen while implementing the Cooperative Relaying Technique as in Fig. 6.

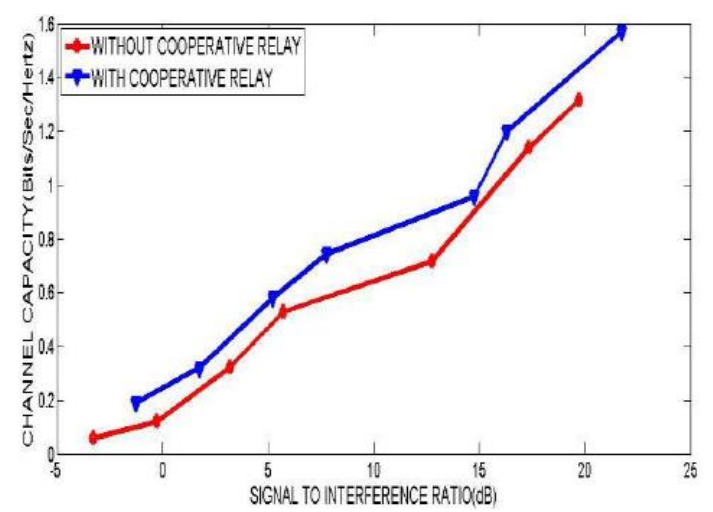

Figure 6. SINR VS CHANNEL CAPACITY(at the primary receiver)

With Cooperative relay the capacity improves by 0.2 bits/sec/Hertz. As the received power improves at the primary receiver due to the amplify and forward type of relay. This in turn improves the SNR which directly enhances the capacity of the system as shown in Fig.7.

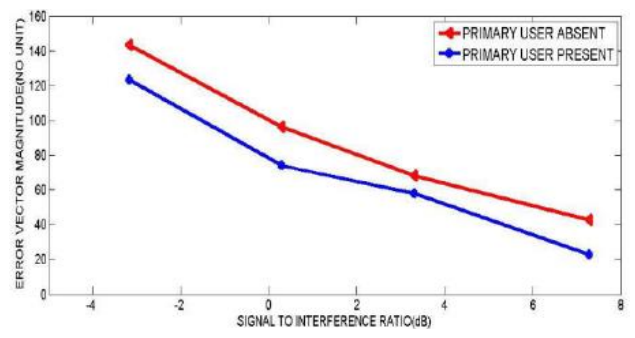

Figure 7. SINR VS ERROR VECTOR MAGNITUDE(at the secondary receiver)
There is an improvement of 20-30 in the EVM using cooperative sensing as the SINR improves due to lesser interference when the primary user is absent. This is shown in Fig. 8.

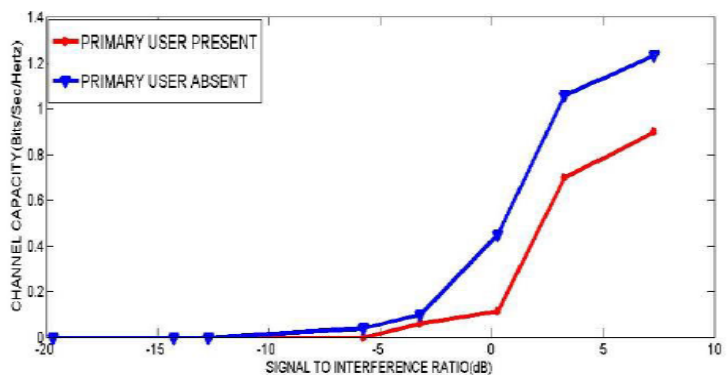

Figure 8. SINR VS CHANNEL CAPACITY(at the secondary receiver)

With Cooperative Sensing the Capacity improves by $0.4 \mathrm{bits} / \mathrm{sec} /$ Hertz as shown in Fig. 9. This is because with cooperative sensing the absence of the primary user is used to full effect by transmitting with full power P0 and this improves the SINR directly leading to the enhancement of the channel capacity.

\section{CONCLUSION AND FUTURE WORKS}

The paper emphasizes on the capacity improvement which is proposed here using cooperative relays and cooperative sensing. The figures represented above prove that along with cooperative localization the interference mitigation can be done more efficiently using these techniques. The paper concludes with a note that the cooperative localization of the primary receivers can be extended to 3D (i.e $\mathrm{z}$ axis also). This becomes useful when the altitude at which the devices are placed is taken into consideration. This will certainly improve the QOS at the receiver when used for interference mitigation. More improvements based on the dynamic nature of the networks with respect to mobility will be included in the future works.

\section{REFERENCES}

[1] Tao Jing, Shixiang Zhu, Hongjuan Li, Xiaoshuang Xing, Xiuzhen Cheng, Yan Huo, Rongfang Bie, and Taieb Znat, "Cooperative Relay Selection in Cognitive Radio Networks", IEEE Transactions On Vehicular Technology, Vol. 64, No. 5, May 2015.

[2] Juncheng Jia,Jin Zhang and Qian Zhang,"Cooperative Relay for Cogntive Radio Networks",IEEE INFOCOM 2009 proceedings.

[3] Thang Van Nguyen, Student Member, IEEE, Youngmin Jeong, Student Member, IEEE Hyundong Shin, Senior Member, IEEE, and MoeZ.Win, Fellow, IEEE, "Least Square Cooperative Localization", IEEE Trans. On Vehicular Technology, Vol. 64, 4 April 2015..

[4] Huifeng Wang, Zhan Gao, Yan Guo, "A Survey of Range-based Localization Algorithms for Cognitive Radio Networks" IEEE, Yuzhen Huang Institute of Communications Engineering PLA University of Science and Technology Nanjing, Email:wanghuifeng1208@163.com, 3 December 2012

[5] Murphy-Heremen "Determination of a position in three dimension using trilateration and approximate distances" MCS- 95-07, Oct 1995. 